\title{
A Modeling and Experimental Method for Accurate Thermal Analysis of AlGaN/GaN Powerbars
}

\author{
Vice Šodan ${ }^{1,2}$, Steve Stoffels ${ }^{1}$, Herman Oprins ${ }^{1}$, Martine Baelmans ${ }^{2}$, Stefaan Decoutere ${ }^{1}$, Ingrid De Wolf ${ }^{1,2}$ \\ ${ }^{1}$ IMEC, Leuven, Belgium \\ ${ }^{2}$ KU Leuven - University of Leuven, B-3000, Belgium
}

\begin{abstract}
In this work we present a novel and comprehensive method for thermal characterization of GaN based transistors. By means of two coupled simulations (TCAD and FEM) a detailed insight into the device physics has been provided whereas an experimental method (low-RF drain conductance measurement) is recognized as a simple and accurate method for a full transient analysis of the transistors. The method (modeling and experiments) has been performed on several different $\mathrm{GaN}$ based transistors where a good agreement between the simulations and experiments is obtained.
\end{abstract}

Keywords-high electron mobility transistor (HEMT), gallium nitride, power dissipation, thermal analysis, finite element analysis, self-heating effect (SHE), thermal impedance

\section{INTRODUCTION}

Heterostructure AlGaN/GaN high electron mobility transistors (HEMTs) have been demonstrated to be promising semiconductor devices for high-power RF and power switching applications [1]. Due to their superior performances $\mathrm{GaN}$ based devices show clear advantages compared with the standard silicon ( $\mathrm{Si}$ ) power components. GaN, as a wide band-gap material $(3.4 \mathrm{eV})$, exhibits a high breakdown field while the polarization effects in the $\mathrm{AlGaN} / \mathrm{GaN}$ heterostructure generate a two-dimensional electron gas (2DEG) with a high carrier concentration $\left(10^{13} \mathrm{~cm}^{-2}\right)$ and respectability high electron mobility $\left(2000 \mathrm{~cm}^{2} / \mathrm{Vs}\right)$. These properties enable the fabrication of $\mathrm{GaN}$ power switching devices with a high breakdown voltage, low on-resistance and high switching frequency. The high power densities enabled by these components can lead to an increased device temperature, as due to Joule heating a substantial amount of heat is generated. This self-heating effect (SHE) is even more pronounced in GaN components where much higher current densities are imposed on smaller volumes, compared to equivalent silicon power devices, leading to higher power densities per area and thus higher junction temperatures. Besides the degraded electrical performances (leakage current, on-resistance, etc.), the reliability of the devices is highly dependent on the SHE effect and the temperature gradients in the junction. Accordingly, to explore the full benefits of $\mathrm{GaN}$ based power components, the junction temperature and global thermal performances should be carefully characterized and controlled in the early design stage.

Several experimental methods for thermal analysis and junction temperature estimation have been reported. Optical methods (infrared imaging [2], Raman spectroscopy [3]) have shown good results with a resolution high enough to capture hot spots. Still, such methods are robust and require physical or visual access to the device surface which makes them impractical for further thermal characterization. Electrical methods [4] use standard measurement equipment where an accuracy of the method is dependent on a calibration process and can result in a junction temperature underestimation [3]. On the other hand, thermal simulations provide a deep insight into the temperature distribution but, due to the assumptions which do not correspond to the exact physical reality, the interpretation of results requires special caution.

For this work we have developed a simple but fairly comprehensive method which accurately models the transient thermal behavior of the GaN power transistors. The method is developed by coupling TCAD simulations (in order to model the exact power distribution profiles) and finite element modeling (for global thermal behavior). Moreover, the simulations have been accompanied by experimental assessment of thermal behavior of GaN transistors. A low-RF extraction method has been used to experimentally determine the output conductance which help us to determine the thermal impedance of transistors. The method has been proven to be accurate for SHE characterization [5] and we have experimentally carried out on $\mathrm{GaN}$ based power devices.

The description of the developed method for the thermal characterization is explained for a single-finger transistor in Section II. The results of the thermal characterization performed on several different GaN power devices are shown in Section III whereas Section IV shows the analysis of large powerbars.

\section{METHOD DESCRIPTION}

\section{A. Finite element electro-thermal modeling}

In order to understand the thermal behavior of $\mathrm{AlGaN} / \mathrm{GaN}$ devices a finite element analysis has been performed. The ambient and boundary conditions that are present during the measurements on a probe station were applied to the models. It is worth noting that the impact of the package is neglected for this work, but can be added afterwards as an additional boundary condition. The simulation process has been developed by coupling transistor level 2D TCAD (Synopsys) simulations with $3 \mathrm{D}$ thermal finite element simulations, in order to improve the model accuracy and physical correctness. The motivation for doing this has been found in the fact that the use of simplified power profiles (such as uniform heating profile below the gate or along the device length) as shown in Fig. 1 results in an underestimation of the peak temperature. The purpose of the TCAD work was to simulate the linear and saturation region of the transistors and to get accurate information about the power dissipation profile along the 


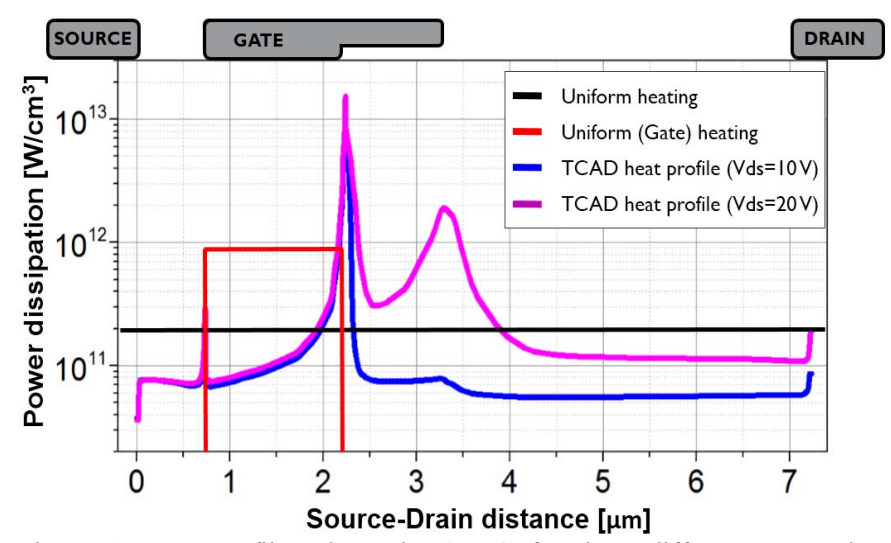

Figure 1- Heat profiles along the 2DEG for three different approaches (uniform heating along the length or below the gate and detailed TCAD approach)

2DEG. The simulation domain in TCAD is limited to $2 \mathrm{D}$ due to the complex computational method and resulting long simulation time. The simulations conditions were matched to correspond to the measurement conditions. Accordingly, a maximum drain current of about $0.5 \mathrm{~A} / \mathrm{mm}$ was used and the output characteristic was simulated for a drain voltage sweep up to $25 \mathrm{~V}$. The power dissipation profiles are extracted from the simulations results taking a line scan along the 2DEG from the source to the drain. The thickness of the 2DEG is assumed to be just a few nanometers [6] after which the concentration of electrons exponentially drops. Thus, power profiles are extracted at $1 \mathrm{~nm}$ below the $\mathrm{AlGaN} / \mathrm{GaN}$ interface. Fig. 1 presents the power dissipation profiles of the device with a gate field-plate. Apart from this configuration, other gate and source field-plate configurations are possible to characterize. Based on our previous work [7] an introduction of more field-plates on top of the gate and active width can improve the thermal behavior of devices and change the shape of the power profile. Looking back at Fig. 1, it is also important to determine the change of power profile with the respect to the drain voltage. Thus, it is not possible to extract a single power dissipation profile but a more detailed approach is required where a selection of power profiles has to correspond to the experimental bias conditions. Once the power dissipation profiles are known they are used as a heat generation condition for the global 3D finite element simulations (MSC MARC) in order to calculate a temperature distribution in the device. The location dependent power profiles (Fig. 1) obtained from the TCAD simulations are positioned between the source and drain in the form of volumetric power density. The models take into account the influence of the wafer. The initial and ambient temperature is $27^{\circ} \mathrm{C}$ while the bottom of the model is kept at the same fixed temperature. To take the heat losses to the ambient into account, convective boundaries conditions are applied at the top side of the model.

The goal of the simulations is capturing the transient thermal response of the characterized transistors. Thus, the temperature distribution in the model is calculated at each time step starting from the smallest time step of $10^{-11} \mathrm{~s}$ up to the steady-state condition. The thermal impedance is calculated from the temperature response by dividing it with the power density $[\mathrm{W} / \mathrm{mm}]$ used in the simulation. The thermal impedance response is this form is used for a comparison with the experimental results. Additionally, an equivalent Foster thermal network consisted of thermal resistances and capacitance can be extracted from this response. The transient thermal impedance in the time domain can be described as

$$
z_{t h}(t)=\sum_{i=1}^{N} R_{i}\left(1-\exp \left(\frac{-t}{R_{i} C_{i}}\right)\right)
$$

where $R_{i}$ and $C_{i}$ are the thermal resistance and thermal capacitances, respectively and $N$ represents the number of terms of equivalent thermal network.

\section{B. Experimental measurements}

Experimental measurements have been performed in order to validate the simulation work but also to investigate an appropriateness of the method (low-RF extraction method) for the static and dynamic thermal characterization. The method is based on a small-signal conductance $\left(\mathrm{g}_{\mathrm{ds}}\right)$ measurement where a small AC signal is superimposed at a selected bias point of the transistor. The measured conductance at the bias point shows a strong dependence on the frequency of the AC signal. At low frequencies the device temperature is able to follow slow AC signal, while at high frequencies the AC signal is too fast and the temperature is not able to follow it any more. This fact results in a variable (not constant) output conductance as a function of frequency depicted on Figure 2a. The measurements are performed using a HP4294A Impedance Analyzer in a frequency range from $40 \mathrm{~Hz}$ up to $6 \mathrm{MHz}$. The real part of the thermal impedance of the measured transistor according to [10] can be calculated as

$$
\operatorname{Re}\left(Z_{t h}\right)=\frac{g_{d s, D C}-g_{d s, A C}}{\frac{d I_{d s}}{d T_{a m b}} \cdot\left(I_{d s}+V_{d s} \cdot g_{d s, D C}\right)}
$$

where $g_{d s, D C}$ is the output conductance at the low frequencies, $g_{d s, A C}$ is the output conductance at the high frequencies, $I_{d s}$ and $V_{d s}$ are current and voltage at the bias point. The coefficient $d I_{d s} / d T_{a m b}$ shows the influence of the ambient temperature on the drain current and can be measured on a hot chuck. Towards getting a full transient response it is necessary to estimate the thermal capacitances not just thermal resistances. Pre-existing works dealing $[5,8,9]$ with this extraction method were focused on the extraction of one thermal capacitance by empirically determining one time constant from the measured response. In this work an extraction of the full transient response is based on the fact that the measured real part of the thermal impedance (Eq.2) contains the information not just about the total steady-state thermal resistance but also the info about the transient behavior. This fact can be verified if the thermal impedance in the frequency domain (for simplicity only one $\mathrm{RC}$ term shown) is parsed on the real and imaginary part as follows

$$
z_{t h}(j \omega)=\frac{R}{1+(\omega R C)^{2}}-j \frac{\omega R^{2} C}{1+(\omega R C)^{2}}
$$




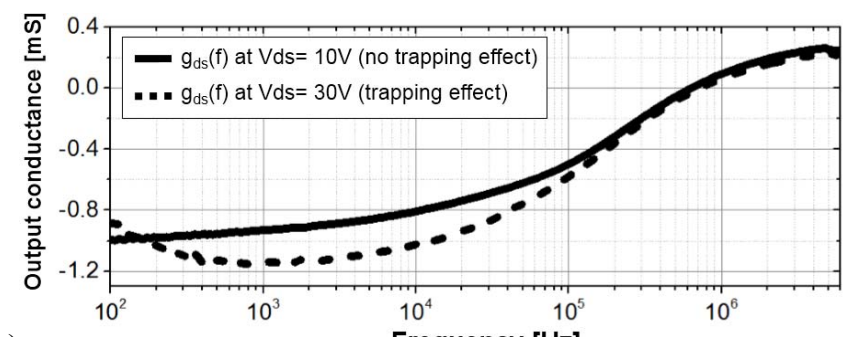

a)

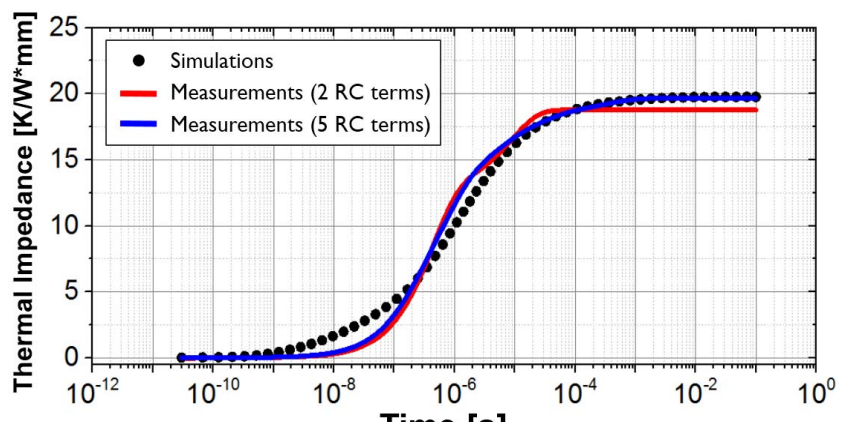

b)

Time [s]

Figure 2- Thermal analysis of single-finger transistor $(1 \times 100 \mu \mathrm{m})$ a) Measured output conductance in the frequency domain, b) Comparison between simulations (dots) and time domain RC representation of the extracted $\mathrm{R}$ and $\mathrm{C}$ values of the measured result in the frequency domain (line)

Thus, the measured drain conductance curve has been transformed in the curve which correspond to the real part of thermal impedance. By doing this we are able to estimate a set of measured RC terms (time constants) which are then plotted in the time domain (Eq.1) and compared with the simulation result (Fig.2b). It is worth noting that GaN HEMTs have a highly expressed self-heating effect with respect to the characterized devices from $[5,8,9]$. In addition, the dimensions are such that dominant thermal time constants are not expected to be lower than $1 \mu \mathrm{s}$ which makes this method easy to apply (no need for S-parameters measurements). Nevertheless, special attention should be given to trapping effects. During the analysis it was observed that for $\mathrm{Vds}>25 \mathrm{~V}$ a trapping effect can occur (Figure 2a, dashed line) which introduces a voltage dependent frequency dispersion at low frequencies unrelated to the thermal effect.

\section{CHARACTERIZATION}

The method verified on the single-gate transistor has been applied on several different GaN HEMTs: $2 \times 100 \mu \mathrm{m}$, $2 \times 200 \mu \mathrm{m}$ and $6 \times 200 \mu \mathrm{m}$ transistors. For the latest one,

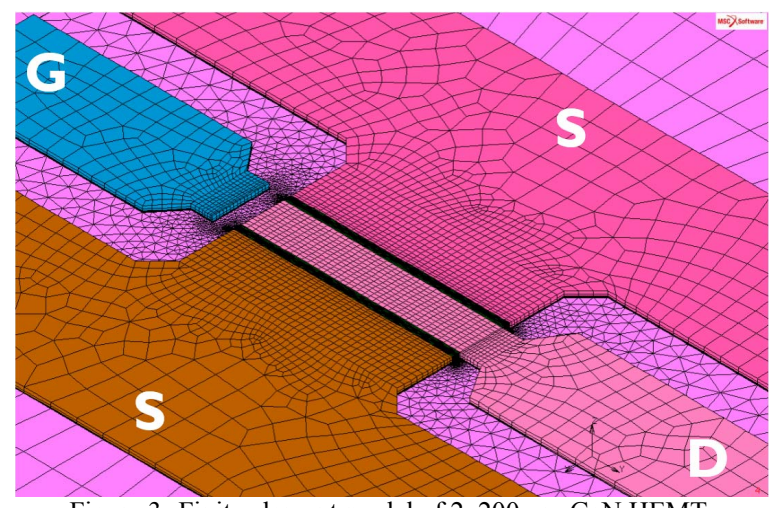

Figure 3- Finite element model of $2 \times 200 \mu \mathrm{m}$ GaN HEMT

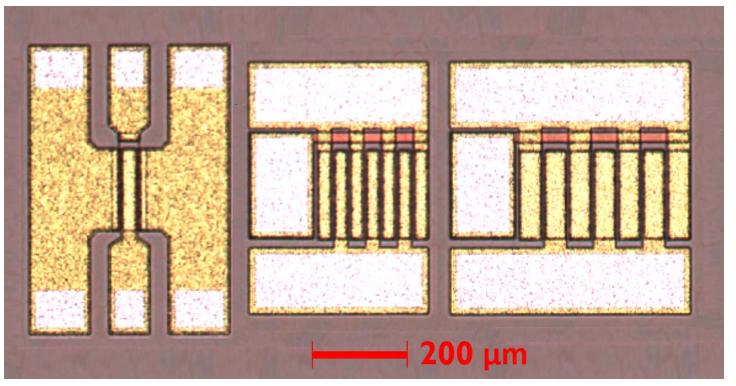

Figure 4- GaN HEMTs on wafer (from left to the right): $2 \times 200 \mu \mathrm{m}$, $6 \times 200 \mu \mathrm{m}$ (30 $\mu \mathrm{m}$ finger spacing) and $6 \times 200 \mu \mathrm{m}(50 \mu \mathrm{m}$ finger spacing) GaN HEMT

different gate-to-gate spacing values between fingers has been considered. Accordingly, the corresponding models have been developed. The model of $2 \times 200 \mu \mathrm{m}$ transistors is shown in Fig. 3 while Fig. 4 shows some of the measured devices. In general, the characterization depends on a power level $\left(P_{d i s s}=I_{d s} \cdot V_{d s}\right)$ at which the experiments and measurements are performed. Due to the non-linear dependency of the thermal conductivities on the temperature the thermal resistance in the steady-state may increase as a function of power. Thus, we carried out the analysis on a constant power level of $2.5 \mathrm{~W} / \mathrm{mm}$. Fig. 5 presents the results for the $2 \times 100 \mu \mathrm{m}$ transistor and the twice as large $2 \times 200 \mu \mathrm{m}$ transistor. The measured conductance curve is shown on the top while the thermal impedance (dots for the simulations, lines for the measurements) are shown below. For simplicity, we only show the fitting curves for three $\mathrm{RC}$ terms. Of course, a larger number of terms means potentially better fit but the complexity increases. As a general impression from the figures a mismatch between simulated and measured thermal responses can be

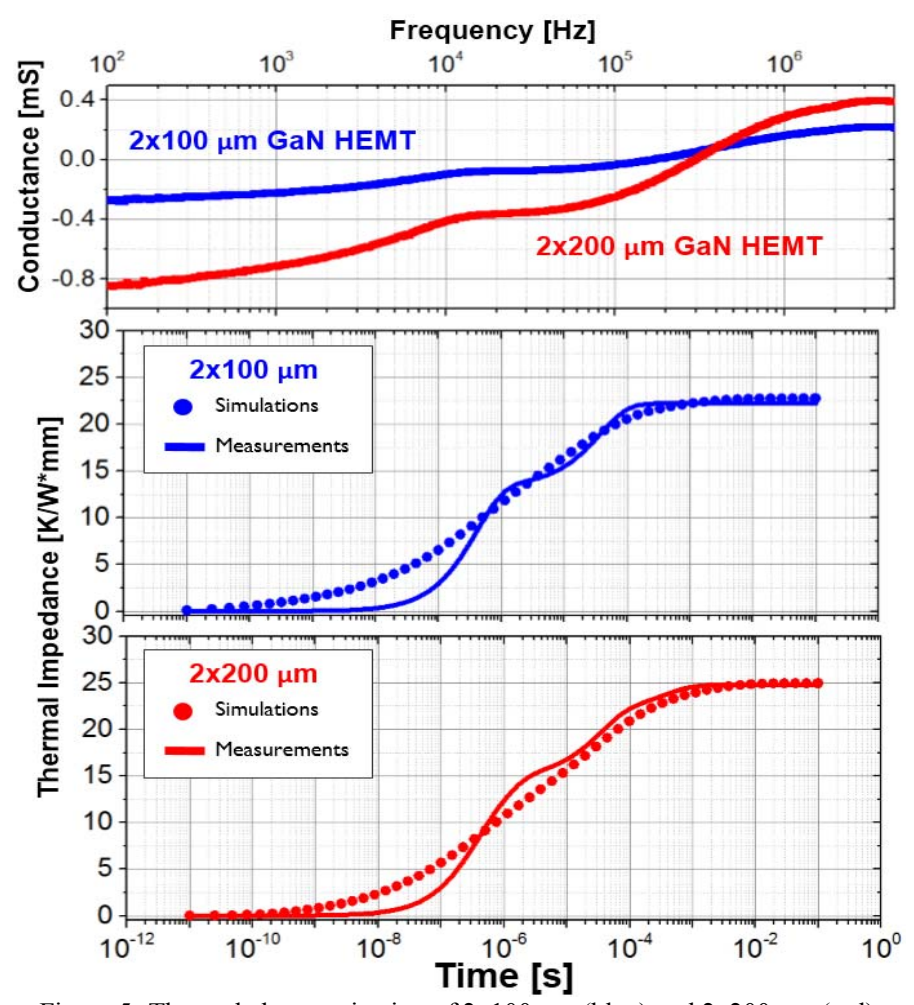

Figure 5- Thermal characterization of $2 \times 100 \mu \mathrm{m}$ (blue) and $2 \times 200 \mu \mathrm{m}$ (red) GaN HEMTs 

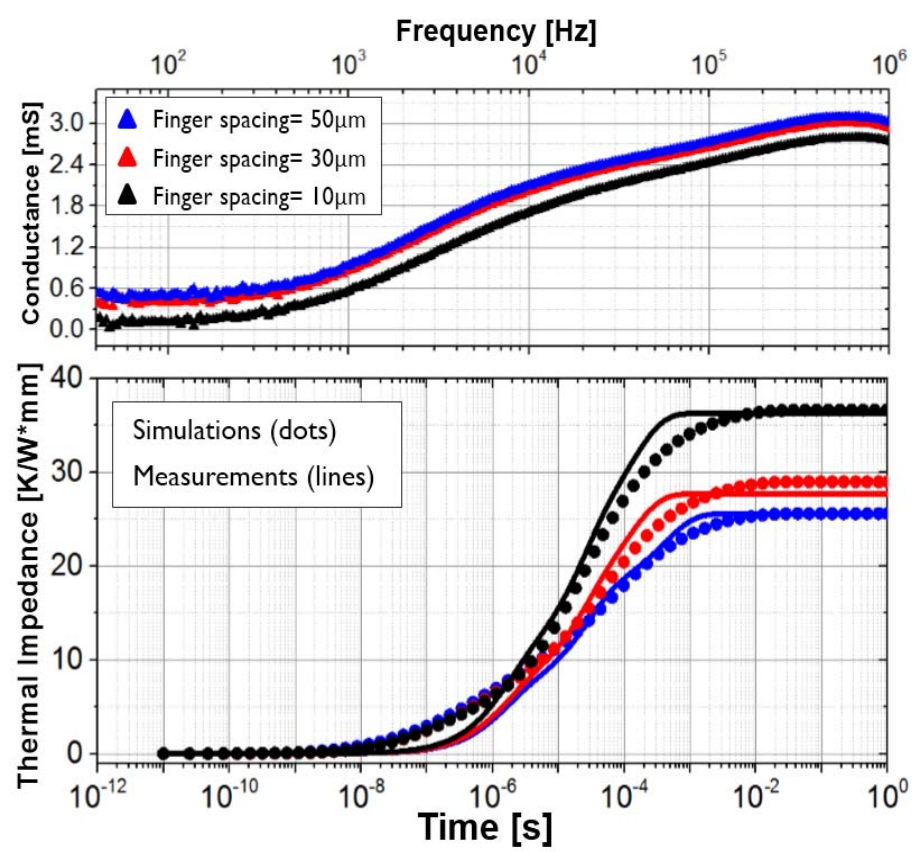

Figure 6- Thermal characterization of $6 \times 200 \mu \mathrm{m}$ transistor with different finger spacing: $10 \mu \mathrm{m}$ (black), $30 \mu \mathrm{m}$ (red) and $50 \mu \mathrm{m}$ (blue)

observed for the smallest time steps (the time steps between $10^{-10} \mathrm{~s}$ and $10^{-6} \mathrm{~s}$ ). Due to the limitation of the measurement setup (the frequency sweep up to $6 \mathrm{MHz}$ ) the smallest time constants we could capture were around $1.2 \cdot 10^{-6} \mathrm{~s}$, explaining the discrepancy between measurements and simulations below this value. Accordingly, increasing the number of terms does not contribute to a better fitting in this case. This mismatch is less expressed as the size of devices increases, as all time constants are longer for this case. The analysis was also extended to the multi-finger devices with three different gate-to-gate (finger) spacing versions (Fig.6). As is shown in Fig.5 and Fig.6 the different thermal behavior of the characterized devices can be observed. Fig.5 shows the differences with respect to the device active width (i.e. finger length) while Fig.6 shows the impact of different finger spacing on the thermal behavior of multi-finger devices. Thus, we have shown that our model can capture the thermal behavior of $\mathrm{GaN}$ transistors under a whole set of design variations (i.e. for different number of fingers, gate to gate pitches and finger lengths). It has all properties necessary to

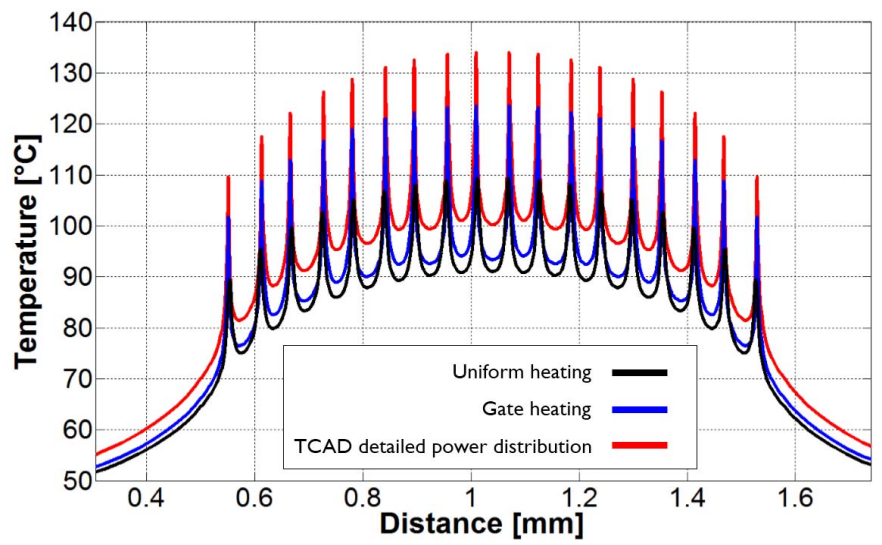

Figure 7- Thermal analysis (simulations) of $18 \mathrm{~mm} \mathrm{GaN}$ powerbar with clear evidence of the peak temperature underestimation for non-TCAD heat profiles perform an accurate design of large $\mathrm{GaN}$ based powerbars.

\section{MODELING POWERBARS}

Once the modeling workflow for thermal characterization has been calibrated and verified it can be used for thermal analysis of large powerbars. Fig. 7 shows the temperature distribution across the fingers of $18 \mathrm{~mm} \mathrm{GaN} \mathrm{powerbar} \mathrm{for} \mathrm{the}$ three different heat generation profiles (from Fig.1). Fig.7 shows the clear evidence of the claim at the beginning that the use of simplified power profiles result in an underestimation of the peak temperature. In this case, an underestimation of $20 \%$ of the peak temperature compared to the accurate power profile is observed. This evidence indisputably highlights the importance of the use of the detailed approach.

\section{CONCLUSION}

In this work we have developed a novel and comprehensive method for the transient thermal characterization and investigation of the self-heating effect in GaN HEMTs. We have shown that the use of simplified power profiles results in significant underestimation of the device temperature. The experimental method has been pointed as a simple and accurate method for the transient thermal analysis which shows a very good agreement with the modeling work under a whole set of design variations. As a result of the presented method, an extracted and validated thermal network can be easily used as a thermal sub-circuit for large-signal compact modeling.

\section{REFERENCES}

[1] N. Ikeda, et al., "GaN Power Transistors on Si Substrates for Switching Applications," Proceedings of the IEEE, Vol. 98, No. 7, July 2010, pp. 1151-1161

[2] Jason A. Carter, Jeremy Acord, Daniel Hoffmann, Andrew Trageser, Charles Page, 'Thermal Factors Influencing the Reliability of GaN HEMTs", 28th Annual IEEE Semiconductor Thermal Measurement and Management Symposium (SEMI-THERM), March 2012, San Jose, CA

[3] Simms R.J.T., Pomeroy J.W., Uren M.J., Martin Trevor, Kuball. M., "Channel Temperature Determination in High-Power AlGaN/GaN HFETs Using Electrical Methods and Raman spectroscopy", IEEE Transactions on Electron Devices, Vol. 55, no. 2, February 2008

[4] Kuzmik J., et al, "Determination of Channel Temperature in AlGaN/GaN HEMTs Grown on Sapphire and Silicon Substrates Using DC Characterization Method", IEEE Transactions on Electron Devices, Vol. 49, no. 8, August 2002

[5] J. Wei, L. Weidong, S.K.H. Fung, P. C.H. Chan, H. Chenming, "SOI thermal impedance extraction methodology and its significance for circuit simulation," in IEEE Transactions on Electron Devices, Vol. 48, No. 4, 2001, pp. 730-736

[6] O. Ambacher, et al., "Two dimensional electron gases induced by spontaneous and piezoelectric polarization in undoped and doped AlGaN/GaN heterostructures," in Journal of Applied Physics, Vol. 87, No. 1, 2000, pp. 334-344

[7] V. Šodan, H. Oprins, S. Stoffels, M. Baelmans, I. De Wolf,” Influence of field-plate configuration on power dissipation and temperature profiles in $\mathrm{AlGaN} / \mathrm{GaN}$ on Silicon HEMTs", in press.

[8] A. J. Scholten, et al.," Experimental assessment of self-heating in SOI FinFETs, " IEEE International Electron Devices Meeting (IEDM), 2009

[9] S. Makovejev, S.Olsen, J.-P. Raskin, "RF extraction of Self-Heating Effects in FinFETs", IEEE Transactions on Electron Devices, 2011 